and enhanced. Finally, in addition to the mandatory training all trainees receive, the taught material during induction should be tailored more towards new doctors' needs.

\section{JUNIOR DOCTORS AS LEADERS IN DEVELOPMENT AND LIVE MANAGEMENT OF A CONTINGENCY ROTA DURING THE COVID-19 PANDEMIC: OUR EXPERIENCE AT THE GENERAL SURGERY DEPARTMENT IN QUEEN ELIZABETH HOSPITAL, LONDON}

Michail Chatzikonstantinou, Cesar Brito-Ramirez, Paolo Sorelli. SR in General and UGI Surgery. HE London, UK

\subsection{6/leader-2020-FMLM.124}

Clinical Fellow, Queen Elizabeth Hospital, London, UK

Consultant Colorectal Surgeon and Clinical Director, Queen Elizabeth Hospital, London, UK

The COVID-19 pandemic has affected millions of patients around the world. Hospital departments had to adapt their services and expand their bed capacity. Our aim was to lead a team that will create a contingency rota in order to anticipate possible COVID-19 related sickness and support front-line specialities, such as Acute Medicine and Intensive Care.

The team involved in the creation of this rota was led by one junior doctor from every grade. Data from the surgical take showed that the average number of daily surgical inpatients dropped from 47 in February, to 22 by the first week of April. This reduction, together with cancellation of elective operations, allowed us to create a contingency plan with a ward cover, an on-call and a stand-by team at all times. We managed to release doctors to support other departments, ensuring that surgical inpatients were receiving the preCOVID-19 standards of care. We, also, created a 'buddy system', predicting possible COVID-19 sickness in the on-call or the ward-cover team. On this contingency rota, there was a ward cover team with one SHO, three FY1s and two registrars, including the team for ITU support and a 'standby' ward-cover team. Two of the FY1 doctors were redeployed to reinforce Acute Medicine. There was constant feedback via a 'WhatsApp' group from the on-call and the ward-cover team to recruit help from the standby team

All doctors who were part of this rota were invited to provide feedback via a satisfaction survey. Out of 13 responses, $61.54 \%$ replied that they were satisfied or very satisfied with the contingency rota, and $76.92 \%$ replied that the rota was fair to very fair. The rota was designed by Junior doctors proving that well-thought planning measures can make all the difference when facing extremely difficult and unprecedented situations like the COVID-19 pandemic.

\section{Quality improvement}

\section{IMPROVING FLUID PRESCRIPTIONS FOR INPATIENT SURGICAL INPATIENTS WITH DIABETES MELLITUS}

Piriyanga Kesavan*, Alexander Light, Oliver Bloom. Bedford Hospital, Bedfordshire Hospitals NHS Foundation Trust, Bedford, UK

10.1136/leader-2020-FMLM.125
Aims Surgical inpatients with diabetes mellitus are common. We aimed to assess the diabetes management of diabetic adult surgical inpatients. This includes reviewing appropriate medication adjustment with altered eating statuses; fluids prescribed alongside a variable rate intravenous insulin infusion (VRIII); numbers of hypo- and hyper-glycaemic events in those on diabetic treatment and appropriate hypoglycaemia management options prescribed.

Methods We audited current performance against national guidelines from he Joint British Diabetes Societies Inpatient Care Group. A prospective snapshot audit was conducted on surgical patients with diabetes mellitus on 3 surgical wards. Data, including diabetic status, eating status, prescriptions and hypo- and hyper-glycaemic events, were collated by reviewing patient notes, feeding instructions and prescription charts. The results were presented at the surgical governance meeting, including a short teaching session, following which a prospective re-audit was conducted.

Results 65 patients were included in the first cycle and 34 in the second. The percentage of patients on gliclazide with a bedtime snack prescribed increased significantly from $28.6 \%$ to $81.8 \%(\mathrm{p}<0.005)$. The percentage of patients with hypoglycaemic and hyperglycaemic events decreased but there was no improvement in the VRIII fluid and PRN hypoglycaemia prescriptions.

Conclusions Robust prescription of diabetic medications and fluids is essential for positive outcomes. The significant increase in bedtime snack prescribing for patients on gliclazide was notable progress. However, there is still more to be improved, with the need for greater awareness of the appropriate VRIII fluid prescription and use of PRN hypoglycaemia management protocol. Continual assessment and improvement of diabetic management is recommended to ensure high quality and cost-effective care.

\section{Understanding leadership through research}

\section{ARE WE GOING IN THE SAME DIRECTION: DO STAFF PERCEPTIONS OF THEIR OWN AND ORGANISATIONAL GOALS ALIGN?}

${ }^{1,2}$ Stuart Bullock, ${ }^{1}$ Chris Turner, ${ }^{2}$ Anne Slowther. ${ }^{1}$ University Hospitals Coventry and
Warwickshire, UK: ${ }^{2}$ Warwick Medical School, UK 10.1136/leader-2020-FMLM.126

Background When we consider what the right thing to do is, we call upon our own morals, professional guidelines, the law and other perspectives. We often combine these with our perceptions of the desires of those in authority. This work aimed to unpack staff perceptions of organisational drivers and gain an understanding of how and where we strike the balance between these multiple (and sometimes conflicting) perspectives.

Method 'Sheila is in the Emergency Department (ED) waiting to be admitted to a bed with a higher level of monitoring. She is about to breach the 12-hour NHS target and a nonmonitored ward bed has become available. You must decide whether or not she can be admitted.' 112 healthcare professionals were asked how they would respond to this scenario. 
They were asked if this was different to what they should do, and what they believed the department and trust management would like them to do.

Results 91\% would keep the patient in ED and 96\% felt their decision was also what they should do. Justifications were consideration of the patient's clinical needs and safety. 68\% believed the department would like them to keep the patient in ED and this dropped to $27 \%$ when asked what trust management would like. The justifications for admitting the patient were avoiding a breach of targets and fears of hospital fines.

Conclusion This scenario saw participants abandon a targetbased approach in favour of doing what they felt was right for a patient. For most there was a conflict between their actions and what they perceived those in positions of authority would want them to do. It is unlikely that those in managerial positions would willingly risk patient safety, but that appears to be the perception of frontline staff. Reductionist messages around targets have the potential to cause dissonance when the message competes with staff values. A more nuanced understanding of the drivers for all staff has the potential to reduce moral distress, a precursor of burnout.

\section{Leading innovation and improvement}

\section{INCREASING ENGAGEMENT IN STUDENT FEEDBACK IN THE CLINICAL YEARS}

${ }^{1}$ Omar Mostafa*, ${ }^{2}$ Amman Malik, ${ }^{1}$ Haroon Shah. ${ }^{1}$ Fifth Year Medical Student, College of Medical and Dental Sciences, University of Birmingham, Birmingham, UK; ${ }^{2}$ Fourth Year Medical Student, College of Medical and Dental Sciences, University of Birmingham, Birmingham, UK

\subsection{6/leader-2020-FMLM.127}

Background At the University of Birmingham, cohorts' student representatives ('Academic Representatives' - AR) are viewed as the intermediaries between staff and students; they represent views of the cohort, express concerns and work closely with the staff to tackle issues across all aspects of the medicine programme, academic and wellbeing. Obtaining feedback is therefore instrumental in identifying student concerns and views and relaying them to the staff.

While this is simpler in pre-clinical years where students are primarily at the university, obtaining feedback becomes difficult in the clinical years due to students being placed at various hospital sites across the West Midlands. In the academic year 19/20, students in Year 4 had their feedback collected using feedback forms distributed electronically by the Year 4 AR. In contrast, the Year 3 cohort had their views represented in the form of designated hospital representatives which fed back to the Year 3 AR.

Aim To assess the effectiveness of using hospital representatives in representing feedback compared with feedback forms.

Method For Year 4, we used the number of feedback responses registered throughout the academic year. For Year 3, we relied on the qualitative responses gained from hospital representatives, alongside feedback from senior medical school staff.
Results For feedback forms, there were 65 responses (cohort of 340 ) in the $19 / 20$ academic year. This is markedly lower than the 185 responses recorded in 15/16 academic year. For hospital representatives, the senior staff found the feedback provided to be more in-depth, practical and purposeful. Also, students found engagement with the hospital representatives more welcoming than using feedback forms.

Conclusion Deployment of hospital representatives across different placement sites has proved to be more efficacious and practical. We recommend this to be implemented on a larger scale across other avenues.

\section{INSPIRING THE FUTURE GENERATION IN PAEDIATRICS DURING THE PANDEMIC - GREAT ORMOND STREET HOSPITAL SUMMER SCHOOL 2020}

${ }^{1}$ Shuang Liu, ${ }^{1}$ Jonathan Smith, ${ }^{2}$ Abbie Tutt, ${ }^{1}$ Justin Poisson, 'Simon Blackburn, ${ }^{1}$ Cristine Sortica da Costa. 'Great Ormond Street Hospital. Post Graduate Medical Education Centre; ${ }^{2}$ The University of Warwick

\subsection{6/leader-2020-FMLM.128}

Introduction Great Ormond Street Hospital (GOSH) Summer School aims to inspire medical students and junior doctors to follow a career in Paediatrics, especially given the low competition ratio in Paediatrics training applications in the last three years.

This year we had to move the conference to a virtual environment due to the outbreak of COVID-19.

Issues We needed to modify the program to be deliverable as a series of webinars. The Summer School's capacity was increased to 500 participants (Virtually) from 75 (face to face).

We explored ways to maintain interactivity, keep engagement, and fulfill our aim to inspire with a much larger group within the virtual environment. Furthermore, we refined our program to reflect the current pandemic.

Interventions To reduce digital fatigue, we changed the length of the conference from 2.5 long days to 4 short days. Each day consisted of 3 or 4 webinars and a career panel session. The career panel sessions focus on sharing the individual journey, which was met with great enthusiasm from the participants.

We utilized multiple tools to facilitate the interaction with the participants and speakers: Slido ( $Q$ and $A$, poll and quiz), a conference App, social media, and the 'Raise Hand' tool within Zoom.

Measurements The conference was well attended with zoom data showed 390, 282,305, and 324 along the four days. Slido showed a high level of engagement with a total of 1005 questions posted and 434 active participants. 344 completed the post-conference survey, $80 \%$ voted the conference being excellent, and $100 \%$ voted for the continuation of delivering the conference online next year.

Lessons Learnt COVID-19 compelled us to run our conference digitally, however we have gained a great deal of experience in management, leadership, and education. We learned the necessity of making rapid decisions and flexible leadership to enable ourselves to deliver quality education during the pandemic. 